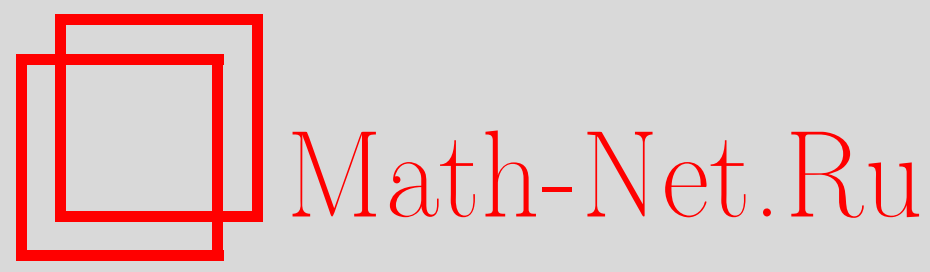

P. Н. Бояринов, О распределении абсолютных значений тригонометрической суммы, Дискрет. матем., 2012, том 24, выпуск 1, 26-29

DOI: https://doi.org/10.4213/dm1169

Использование Общероссийского математического портала Math-Net.Ru подразумевает, что вы прочитали и согласны с пользовательским соглашением http://www.mathnet.ru/rus/agreement

Параметры загрузки:

IP : 3.85 .5 .30

26 апреля 2023 г., 15:05:36 
УДК 519.2

\title{
О распределении абсолютных значений тригонометрической суммы
}

\author{
() 2012 г. Р. Н. Бояринов
}

Доказана теорема о распределении больших значений модуля тригонометрической суммы с лакунарными последовательностями натуральных чисел.

В 1956 г. А. Г. Постников в [1] доказал следующую теорему о распределении значений показательной тригонометрической суммы.

Теорема 1. Пусть

$$
S(\alpha)=\sum_{x=0}^{P-1} e^{2 \pi i \alpha g^{x}}
$$

где $0 \leqslant \alpha \leqslant 1, g-$ челое число, $g \geqslant 2$. Интервал $[0,1)$ эффективно разбивается на два подмножества $\mathfrak{M}_{1}$ u $\mathfrak{M}_{2}$, причем если $\alpha \in \mathfrak{M}_{1}$, то

$$
|S(\alpha)| \leqslant C(\varepsilon) \frac{P}{\ln ^{1 / 2-\varepsilon} P}, \quad 0<\varepsilon<1 / 2,
$$

$u$

$$
\operatorname{mes} \mathfrak{M}_{2}=O\left(e^{-a(\ln P)^{3}}\right)
$$

где а есть некоторая положительная постоянная.

Настоящая заметка посвящена обобщению результата А. Г. Постникова на случай лакунарной последовательности натуральных чисел. Справедлива следующая теорема.

Теорема 2. Пусть дань лакунарнье последовательности натуральных чисел $F_{j}(x)$, $1 \leqslant j \leqslant k$, то есть такие, что для любого $x \geqslant 1$ выполняются неравенства

$$
\frac{F_{j}(x+1)}{F_{j}(x)} \geqslant \beta_{j}>1,
$$

где $x, j, k$ - натуральные числа. Рассмотрим тригонометрическую сумму

$$
S_{k}(\bar{\alpha})=S_{k}\left(\alpha_{1}, \ldots, \alpha_{k}\right)=\sum_{x=1}^{P} e^{2 \pi i\left(\alpha_{1} F_{1}(x)+\ldots+\alpha_{k} F_{k}(x)\right)} .
$$


Для любого $\lambda>0 k$-мерный куб $[0,1]^{k}$ разбивается на два непересекаюшихся подмножества $\mathfrak{M}_{1}(\lambda)$ и $\mathfrak{M}_{2}(\lambda)$. Множество $\mathfrak{M}_{1}(\lambda)$ определяется как множество тех $\bar{\alpha} \in[0,1]^{k}$, для которых $\left|S_{k}(\bar{\alpha})\right| \leqslant \lambda \sqrt{P}$, а $\mathfrak{M}_{2}(\lambda)=[0,1]^{k}-\mathfrak{M}_{1}(\lambda)$. Тогда для меры второго множества $\mathfrak{M}_{2}(\lambda)$ справедлива оченка сверху

$$
\operatorname{mes} \mathfrak{M}_{2}(\lambda)<\exp \left(1-\varkappa \lambda^{2}\right),
$$

где

$$
\begin{aligned}
& \varkappa=\frac{\beta-1}{e \beta}, \\
& \beta=\max _{1 \leqslant j \leqslant k} \beta_{j} .
\end{aligned}
$$

Замечание 1. При $k=1$ и $\lambda=(\ln P)^{3 / 2}$ теорема 2 представляет собой улучшение результата А. Г. Постникова, а именно, что $|S(\alpha)| \leqslant \sqrt{P \ln ^{3} P}$ для всех $\alpha \in[0,1]$ за исключением множества точек $\alpha$, мера которого не превосходит величины $\exp \left(1-\varkappa \lambda^{2}\right)$, где $\varkappa=(g-1) /(e g)$.

Замечание 2. Теорема 2 верна и для суммы

$$
W_{k}(\bar{\alpha})=\sum_{x=1}^{P} e^{2 \pi i\left(\alpha_{1} m_{1} F_{1}(x)+\ldots+\alpha_{k} m_{k} F_{k}(x)\right)},
$$

где $m_{1}, \ldots, m_{k}$ - целые числа, не все равные нулю. В этом случае

$$
\begin{aligned}
& \varkappa=\frac{\gamma-1}{e \gamma}, \\
& \gamma=\max _{1 \leqslant j \leqslant k, m_{j} \neq 0} \beta_{j} .
\end{aligned}
$$

Замечание 3. Теорема 2 верна и для сумм вида $\operatorname{Re} S_{k}(\bar{\alpha}), \operatorname{Im} S_{k}(\bar{\alpha})$. В этом случае

$$
\begin{aligned}
& \varkappa=\frac{\beta-1}{e \beta}, \\
& \beta=\max _{1 \leqslant j \leqslant k} \beta_{j} .
\end{aligned}
$$

Замечание 4. Теорема 2 верна и для сумм вида $\operatorname{Re} W_{k}(\bar{\alpha}), \operatorname{Im} W_{k}(\bar{\alpha})$. В этом случае

$$
\begin{aligned}
\varkappa & =\frac{\gamma-1}{e \gamma}, \\
\gamma & =\max _{1 \leqslant j \leqslant k, m_{j} \neq 0} \beta_{j} .
\end{aligned}
$$

Замечание 5. В силу замечания 3 , результаты теоремы 2 для сумм вида $\operatorname{Re} S_{k}(\bar{\alpha})$, $\operatorname{Im} S_{k}(\bar{\alpha})$ при $k=1$ и $\lambda \geqslant(\ln P)^{3 / 2}$ уточняют результаты работы [2].

Доказательство теоремы 2 опирается на нижеследующую лемму.

Лемма 1. Пусть $F(x)$ - лакунарная последовательность натуральных чисел $и$

$$
\frac{F(x+1)}{F(x)} \geqslant \delta>1 .
$$


Тогда для числа решений $r_{n}(P)$ уравнения

$$
F\left(x_{1}\right)+\ldots+F\left(x_{n}\right)=F\left(y_{1}\right)+\ldots+F\left(y_{n}\right)
$$

в натуральных числах $1 \leqslant x_{1}, \ldots, x_{n}, y_{1}, \ldots, y_{n} \leqslant P$ справедлива оченка сверху

$$
r_{n}(P) \leqslant n !\left(\frac{\delta}{\delta-1}\right)^{n} P^{n}
$$

Доказательство леммы можно найти в [3-5].

Доказательство теоремь 2. Рассмотрим систему уравнений

$$
\begin{aligned}
F_{1}\left(x_{1}\right)+\ldots+F_{1}\left(x_{n}\right) & =F_{1}\left(y_{1}\right)+\ldots+F_{1}\left(y_{n}\right) \\
& \ldots \\
F_{k}\left(x_{1}\right)+\ldots+F_{k}\left(x_{n}\right) & =F_{k}\left(y_{1}\right)+\ldots+F_{k}\left(y_{n}\right) .
\end{aligned}
$$

Тогда для числа решений $v_{j, n}(P) j$-го уравнения системы (1)

$$
F_{j}\left(x_{1}\right)+\ldots+F_{j}\left(x_{n}\right)=F_{j}\left(y_{1}\right)+\ldots+F_{j}\left(y_{n}\right)
$$

в натуральных числах $1 \leqslant x_{1}, \ldots, x_{n}, y_{1}, \ldots, y_{n} \leqslant P$ из леммы 1 следует оценка сверху

$$
v_{j, n}(P) \leqslant n !\left(\frac{\beta_{j}}{\beta_{j}-1}\right)^{n} P^{n} .
$$

Тогда для числа решений $J_{k, n}(P)$ системы (1) справедлива оценка

$$
J_{k, n}(P) \leqslant \min _{1 \leqslant j \leqslant k} v_{j, n}(P) \leqslant \min _{1 \leqslant j \leqslant k} n !\left(\frac{\beta_{j}}{\beta_{j}-1}\right)^{n} P^{n}=n !\left(\frac{\beta}{\beta-1}\right)^{n} P^{n},
$$

где

$$
\beta=\max _{1 \leqslant j \leqslant k} \beta_{j}
$$

Для доказательства основного утверждения теоремы 2 применим неравенство А. А. Маркова

$$
\lambda^{2 n} P^{n} \operatorname{mes} \mathfrak{M}_{2}(\lambda) \leqslant \int_{[0,1]^{k}}\left|S_{k}(\bar{\alpha})\right|^{2 n} d \bar{\alpha}=J_{k, n}(P) \leqslant n !\left(\frac{\beta}{\beta-1}\right)^{n} P^{n} .
$$

Отсюда получаем, что

$$
\operatorname{mes} \mathfrak{M}_{2}(\lambda) \leqslant\left(\frac{n \beta}{(\beta-1) \lambda^{2}}\right)^{n} \text {. }
$$

При $\lambda \geqslant 1 / \sqrt{\varkappa}$ возьмем $n=\left[x \lambda^{2}\right]$, где $[x]-$ целая часть числа $x$. Отсюда

$$
\operatorname{mes} \mathfrak{M}_{2}(\lambda) \leqslant e^{-n}<\exp \left(1-\varkappa \lambda^{2}\right) \text {. }
$$

При $0<\lambda<1 / \sqrt{\varkappa}$ справедливы неравенства

$$
\operatorname{mes} \mathfrak{M}_{2}(\lambda) \leqslant 1 \exp \left(1-\varkappa \lambda^{2}\right) \text {. }
$$

Теорема 2 доказана. 


\section{Список литературы}

1. Постников А. Г., Оценка показательной тригонометрической суммы. Известия РАН. Сер. матем. (1956) 70, 661-666.

2. Гапошкин В. Ф., О скорости приближения к нормальному закону распределений взвешенных сумм лакунарных рядов. Теория вероятностей и ее применения (1968) 13, №3, 445-461.

3. Бояринов Р. Н., Чубариков В. Н., О распределении значений функций на последовательности Фибоначчи. Доклады РАН (2001) 379, 9-11.

4. Бояринов Р. Н., Центральная предельная теорема для равномерного распределения дробных долей быстрорастущих последовательностей. Вестник МГУ. Сер. 1, матем., механ. (2001) №5, $52-54$.

5. Бояринов Р. Н., Нгонго И. С., Чубариков В. Н., О новых метрических теоремах в методе А. Г. Постникова. В сб.: Актуальные проблемы теории чисел: Труды IV Международной конф., Тула, 2002, с. 5-31. 